\title{
Accuracy of the measurement with the second order axial gradiometer
}

\author{
I. Šimáček, P. Jurdák, J. Maňka and M. Škrátek \\ Department of Magnetometry, Institute of Measurement Science, Slovak Academy of Sciences, \\ Dúbravská cesta 9, 84104 Bratislava, Slovakia, e-mail: umersima@savba.sk
}

Determining the accuracy of the measurement by using the second order axial gradiometer is presented. Output signals from the SQUID gradiometer system and calculated values of systematic errors depending on the shape and positioning of small cylindrical samples containing magnetized material are introduced. The measurement of the gradiometer detection characteristics and systematic error analysis were accomplished.

Keywords: second order axial gradiometer, magnetic measurement, cylindrical sample, systematic error

\section{INTRODUCTION}

$S^{\mathrm{p}}$ PECIFIC PROPERTIES of gradiometer systems are well known and their advantages are often employed in biomagnetic measurements. One possible area involves research on biological materials in a human body containing various paramagnetic or ferri- alternatively ferromagnetic substances. Using the small cylindrical samples, magnetic properties or concentrations of these substances are frequently investigated. With the help of various SQUID systems with the $2^{\text {nd }}$ order axial gradiometers, the remanent magnetic induction $B_{\mathrm{r}}$ generated by the magnetized sample content is measured [1]. It is known that detection characteristics [2] of that type of gradiometer are relatively narrow, consequently the acquired output data strongly depend on the sample dimensions and its position to the gradiometer. Technical arrangement for the measurement of the gradiometer detection characteristics and calculation of some systematic errors in magnetic measurement of samples with volume up to $12 \mathrm{~cm}^{3}$ are presented. The aim of this work was to investigate the accuracy of the measured data.

\section{SubJect \& Methods}

To evaluate the influence of the shape, volume and position of a measured sample on the accuracy of the observed output data, the following procedure has been made:

i. The powdered $\gamma-\mathrm{Fe}_{2} \mathrm{O}_{3}$ with the mass of $5 \mathrm{mg}$ was inserted into the small cylindrical hole (diameter $d_{1}=$ $1 \mathrm{~mm}$, volume $V_{1} \approx 1 \mathrm{~mm}^{3}$ ), which had been bored in a hardened epoxy matrix.

ii. This miniature sample $S_{1}$ was magnetized for $30 \mathrm{~s}$ in the steady homogeneous magnetic field of $120 \mathrm{mT}$, whereby $\mathrm{S}_{1}$ achieved a remanent magnetic moment $\vec{m}_{r 1}\left(0,0, m_{\mathrm{r} 1}\right)$.

iii. Then $S_{1}$ was positioned in stages on a movable platform at five vertical distances $h$ from the centre of the closest coil of the $2^{\text {nd }}$ order axial gradiometer (baseline length $b_{\mathrm{g}}$ $=4.5 \mathrm{~cm}$, with $d_{\mathrm{g}}=3 \mathrm{~cm}$ diameter coils). Consequently, $\vec{m}_{r 1}$ was oriented along the $z$-axis normal to the planes of the gradiometer pick-up coils, Fig.1. iv. In every position $h, \mathrm{~S}_{1}$ was shifted from the midpoint horizontally at the distances of $x= \pm 15 \mathrm{~cm}$. During the whole movement the output voltage $U_{1}$ was recorded by the one-channel SQUID gradiometer system (white noise level $<30 \mathrm{fT} \mathrm{Hz}^{-1 / 2}$ )

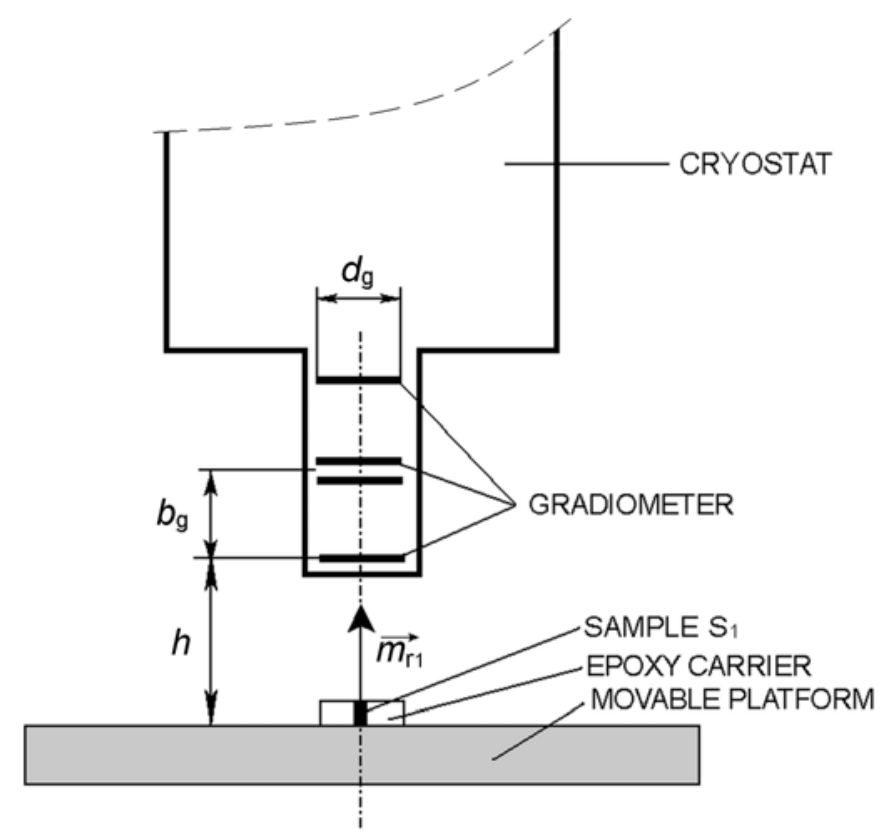

Fig.1 Schematic arrangement for magnetic measurement of the sample $S_{1}$ with the $2^{\text {nd }}$ order axial gradiometer.

Measured values of $U_{1}$ were proportional to $z$ component of the remanent magnetic induction $B_{\mathrm{r}}$ from the magnetized $\mathrm{S}_{1}$. Under assumption that this magnetized mass element represents one magnetic dipole, the gradiometer detection characteristics $U_{1}=f\left(m_{r 1}, x, h\right)$ could be approximately determined. 


\section{RESULTS}

Fig.2 shows the output signals $U_{1}$ induced by horizontal positioning of $S_{1}$ as a function of distances $h=3,4,5,7$ and 9 $\mathrm{cm}$. It is evident that each value of $U_{1}$ is smaller in comparison to $U_{1 \max }$ being measured in the central position of $x_{0}=0$. This difference $U_{1 \max }-U_{1}$ can be defined as a systematic error $e_{1}=$ $\Delta U_{1}$ related to the given gradiometer construction, horizontal position $x$ and applied vertical position $h$ of $\mathrm{S}_{1}$.

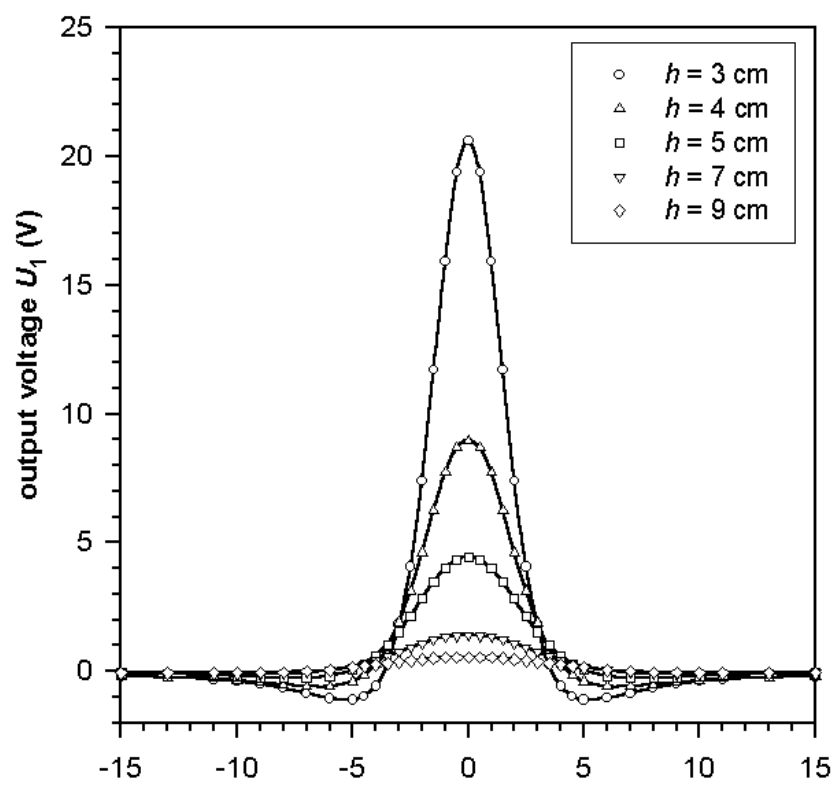

horizontal distance from the central position $x(\mathrm{~cm})$

Fig.2 Experimental gradiometer detection characteristics in response to horizontal position $x$ of the sample $\mathrm{S}_{1}$ being placed at the vertical distances $h$

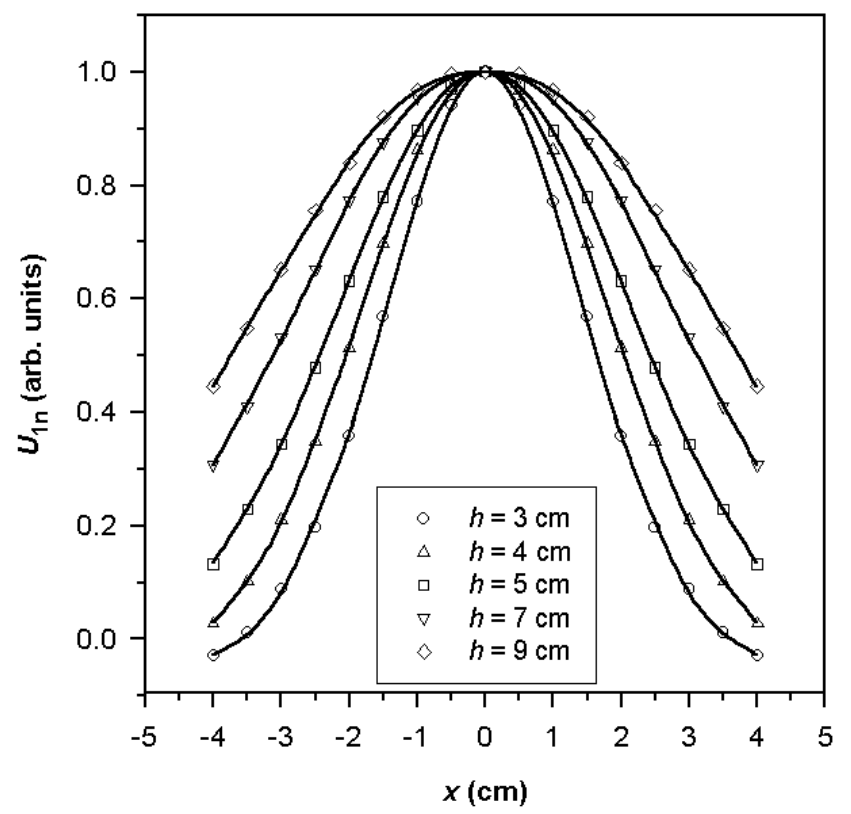

Fig.3 Normalized gradiometer detection characteristics in response to the horizontal position $x$ for various $h$.
The values of $U_{1}$ were normalized with respect to $U_{1 \max }$ and then fitted to $U_{1 \mathrm{n}}$ by the equation

$$
U_{1 n}=y_{0}+a \exp \left(-0.5\left(\frac{x-x_{0}}{b}\right)^{c}\right)
$$

where $a, b, c, x_{0}$ and $y_{0}$ are the parameters of the used fitting. The normalized gradiometer detection characteristics $U_{\text {ln }}$ are plotted in Fig.3.

The first step was aimed at estimating the systematic errors $e_{\mathrm{s}}$ for real larger cylindrical samples $\mathrm{S}$ with the height of $1 \mathrm{~mm}$ and the diameters of $d=1,2,3$ and $4 \mathrm{~cm}$, being gradually placed at the vertical distances of $h=3,4,5,7$ and $9 \mathrm{~cm}$. Assuming that the sample $\mathrm{S}$ is magnetically isotropic and consists of many $S_{1}$ without interactions among them, it can be divided into the set of rings with the widths of $1 \mathrm{~mm}$, Fig. 4.

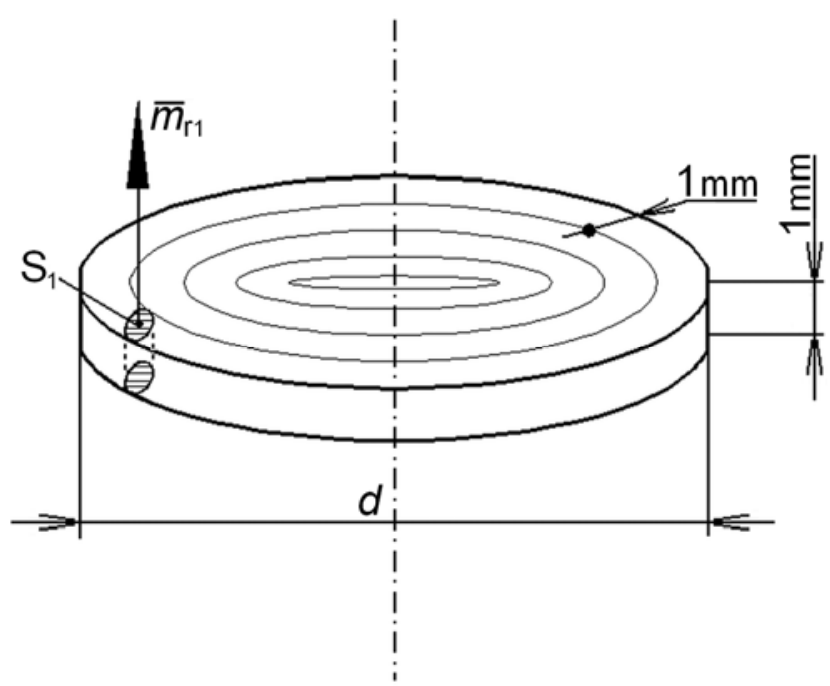

Fig.4 Schematic configuration of the sample $\mathrm{S}_{1}$ divided into the set of rings.

Then the magnitude of the measured total output voltage $U_{\mathrm{s}}$ will be dependent both on the position of each $\mathrm{S}_{1}$ and on the magnitude of the area of the ring which $\mathrm{S}_{1}$ lies on. In the first approximation, $e_{\mathrm{s}}$ can be determined (in percents) by the following equation

$$
e_{s}=100-\sum_{1}^{m} P_{m} U_{1 m n}
$$

where $m$ is the number of rings with the width of $1 \mathrm{~mm}, P_{\mathrm{m}}$ is the ratio of the $m$-ring area to the whole sample's area $P_{\mathrm{c}}$ (also in percents) and $U_{1 \mathrm{mn}}$ is the normalized output voltage derived from (1). Figure 5 shows the corresponding calculation of $e_{s}$ for the cylindrical sample $S$ with the thickness of $1 \mathrm{~mm}$ for four diameters $d$ (in $\mathrm{cm}$ ). Data were fitted with an exponential decay function

$$
e_{s}=y_{0}+a \exp (-b h)
$$




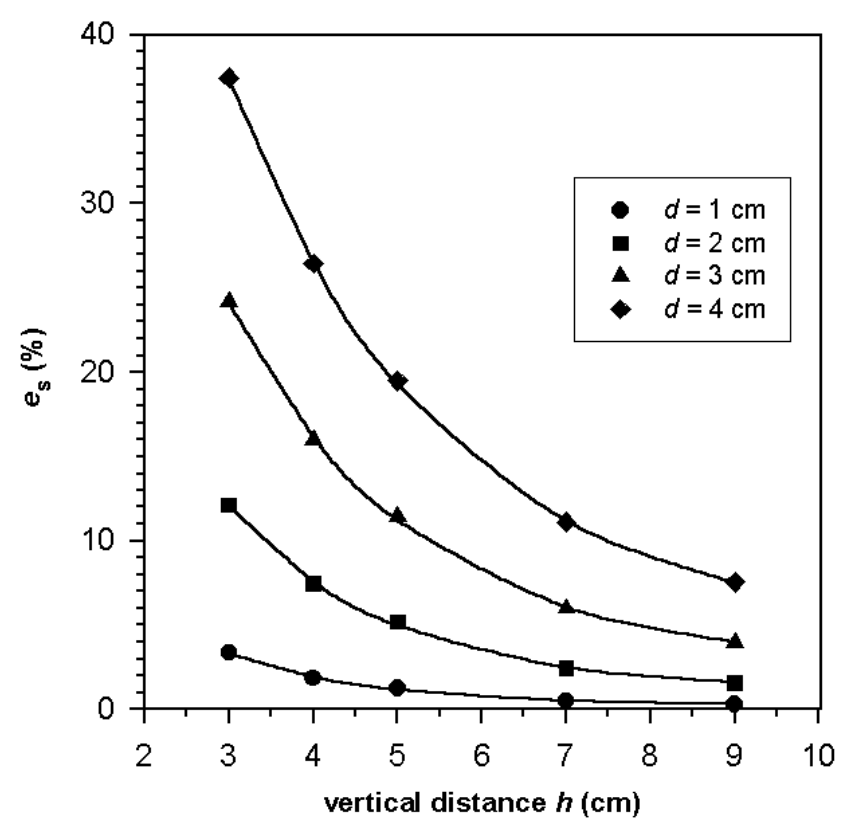

Fig.5 The calculated dependences of $e_{\mathrm{s}}$ on the vertical distance $h$ for samples $\mathrm{S}$ having several diameters $d$.

The resulting $e_{\mathrm{s}}$ expresses how much (in percents) of the measured output voltage $U_{\mathrm{s}}$ falls off in comparison to the output voltage $U_{\text {sm }}$ measured if all $\vec{m}_{r 1}$ of the sample $\mathrm{S}$ were theoretically concentrated in the midpoint $x_{0}=0$. Consequently, the wider the sample and the closer to the gradiometer, the greater $e_{\mathrm{s}}$ is achieved. It can be shown in this example: If $\mathrm{S}$ with $d=4 \mathrm{~cm}$ changes its position from $h=4$ $\mathrm{cm}$ to $9 \mathrm{~cm}, e_{\mathrm{s}}$ decreases approximately five times, specifically, from $37.46 \%$ to $7.45 \%$. Likewise, if S has only $d$ $=1 \mathrm{~cm} e_{\mathrm{s}}$ also decreases from $3.35 \%$ to $0.30 \%$ after the same change of $h$.

In practice, the samples have various heights. Therefore, the total systematic error $e_{\mathrm{sc}}$ for cylindrical samples $\mathrm{S}_{\mathrm{c}}$ with heights $y$ ranging from 1 to $10 \mathrm{~mm}$ was calculated. Using the modified (3) the systematic errors $e_{\mathrm{sy}}$ for each $1 \mathrm{~mm}$ layer of $\mathrm{S}_{\mathrm{c}}$ were determined by the following formula

$$
e_{s y}=y_{0}+a \exp (-b(h-0.1(s-1)))
$$

where $s$ is the appropriate $1 \mathrm{~mm}$ layer measured from the base of the $\mathrm{S}_{\mathrm{c}}$. Then, the final $e_{\mathrm{sc}}$ magnitude can be expressed as the sum of $e_{s y}$ by

$$
e_{s c}=\frac{\sum_{s=1}^{s_{c}} e_{s y}}{s_{c}}
$$

where $s_{\mathrm{c}}$ is the number of $1 \mathrm{~mm}$ layers in the $\mathrm{S}_{\mathrm{c}}$.

The importance of the sample shape for the $e_{\mathrm{sc}}$ magnitude is shown in Fig. 6 illustrating $e_{\mathrm{sc}}$ as a function of $y$ for two samples $\mathrm{S}_{\mathrm{c} 1}$ and $\mathrm{S}_{\mathrm{c} 2}$. These samples have the same volume of $V=1.26 \mathrm{~cm}^{3}$ and contain the same magnetic material but they have different shapes. The first, $\mathrm{S}_{\mathrm{c} 1}$, has dimensions of $d_{1}=4$ $\mathrm{cm}$ and $y=1 \mathrm{~mm}$ and the second one, $\mathrm{S}_{\mathrm{c} 2}$, is with the dimensions $d_{2}=2 \mathrm{~cm}$ and $y=4 \mathrm{~mm}$. In spite of their identical magnetization $M \sim V$, in case of $\mathrm{S}_{\mathrm{c} 1}$ at the distance of $h=4 \mathrm{~cm}$ the calculated $e_{\mathrm{sc}}$ is $26.6 \%$, in comparison to $e_{\mathrm{sc}}=7.64 \%$ for $\mathrm{S}_{\mathrm{c} 2}$. These two identical samples at $h=9 \mathrm{~cm}$ resulted in $e_{\mathrm{sc}}=$ $7.72 \%$ in the first case and $e_{\mathrm{sc}}=1.65 \%$ in the second one.

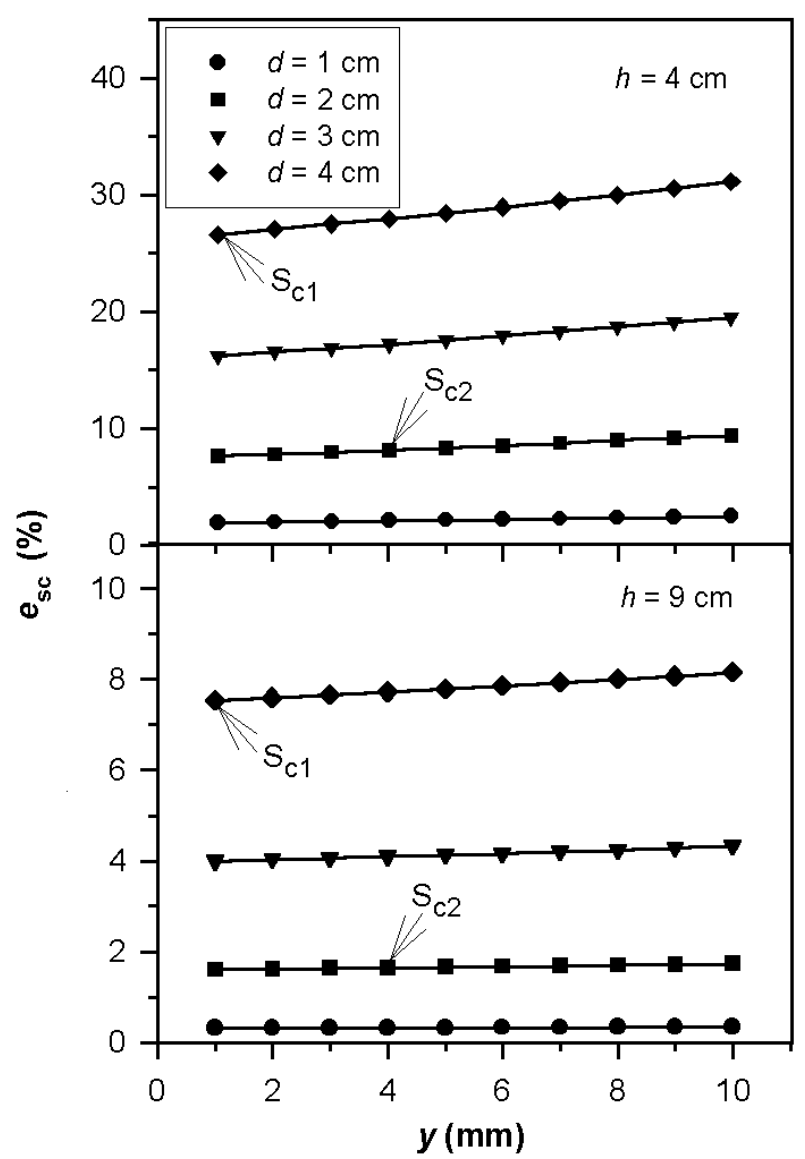

Fig.6 Calculated values of $e_{\text {sc }}$ related to the height $y$ at the distances $h=4 \mathrm{~cm}$ and $9 \mathrm{~cm}$ for four diameters $d$ of the samples $\mathrm{S}_{\mathrm{c}}$.

The effect of various $y$ on $e_{\mathrm{sc}}$ may be also demonstrated by the following example: if dimensions of $\mathrm{S}_{\mathrm{c}}$ are $d=1 \mathrm{~cm}, y=1$ $\mathrm{mm}$ and this sample is located at $h=4 \mathrm{~cm}$, then the calculated $e_{\mathrm{sc}}$ is $1.94 \%$. By increasing $y$ up to $10 \mathrm{~mm}, e_{\mathrm{sc}}$ increases only to $2.5 \%$. For larger $\mathrm{S}_{\mathrm{c}}$ with dimensions of $d=4 \mathrm{~cm}$ and $y=1$ $\mathrm{mm}, e_{\mathrm{sc}}$ rises from $26.60 \%$ to $31.11 \%$ if $y$ enlarges from $1 \mathrm{~mm}$ to $10 \mathrm{~mm}$. Taking into account the sensitivity of the measuring system in terms of accuracy, the samples should be as small as possible, with the shape that is rather higher than wider and placed under the sensor as far as possible.

Of course, the accuracy of the measurement is also affected by the gradiometer construction. From this point of view, $e_{s c}$ decreases if the gradiometer detection characteristics are relatively wide. For example, it can be influenced by increasing of the base length $b_{\mathrm{g}}$. Assuming that the diameters of the gradiometer coils are smaller than the distance $h$, then the relation of $B_{\mathrm{r}}$ between the position of measured magnetic particle can be approximately determined by the following relation [3]. 


$$
\vec{B}_{r}(\vec{m}, \vec{r})=\frac{\mu_{0}}{4 \pi}\left[\frac{3(\vec{m} \cdot \vec{r}) \vec{r}}{r^{5}}-\frac{\vec{m}}{r^{3}}\right]
$$

where $\vec{B}_{r}(\vec{m}, \vec{r})$ is the magnetic induction in the individual gradiometer coils generated from the magnetized particle with the magnetic dipole moment $\vec{m}(0,0, m)$, and $\vec{r}$ is the position vector of that magnetic dipole to the sensing and compensating gradiometer coils. Using (6), Fig.7 illustrates the resulting dependences of $B_{\mathrm{rn}}(x)$ normalized to the largest value, for all defined positions of coils when the base lengths $b_{g}$ are $2.5,4.5$ or $8 \mathrm{~cm}$. One can see that the most flat course has the gradiometer detection characteristic $B_{\mathrm{rn}}(x)$ for $b_{\mathrm{g}}=8$ $\mathrm{cm}$. It means, that the greater $b_{\mathrm{g}}$, the smaller $e_{\mathrm{sc}}$ regardless the sample dimensions or its distance $h$ from the gradiometer.

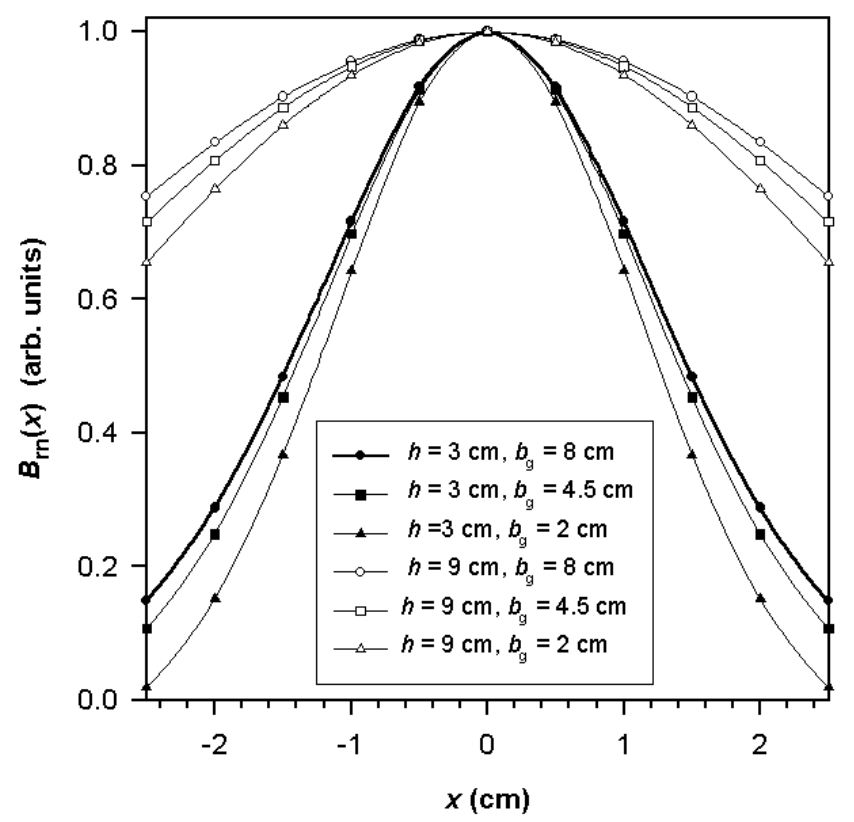

Fig.7 Calculated values of $e_{\mathrm{sc}}$ related to the height $y$ at the distances $h=4 \mathrm{~cm}$ and $9 \mathrm{~cm}$ for four diameters $d$ of the samples $\mathrm{S}_{\mathrm{c}}$.
As shown above, in the measurements of the small-volume samples using $2^{\text {nd }}$ order axial gradiometers it is important to take into account the shape, dimensions and placement of the measured sample. In practice, it is necessary to do some compromises among the used sensitivity in the measuring process, the degree of the noise reduction and the accuracy of the measurement. Thus, the systematic errors should be corrected or have to be taken into account while estimating the standard uncertainty of the used measuring method.

\section{CONCLUSION}

We have presented a type of technique and calculation for assessing the accuracy of the measuring procedure for the given axial gradiometer system. The systematic errors were determined on the basis of the measured detection characteristics of one specific $2^{\text {nd }}$ order axial gradiometer. The measured and calculated data showed that the accuracy strongly depended on the shape and size of the samples and on their position to the gradiometer system.

\section{ACKNOWLEDGMENT}

The work was supported by the Slovak Research and Development Agency, Contract No. APVV-51-059005 and Slovak Grant Agency for Science, Contract No. 2/7084/27.

\section{REFERENCES}

[1] Williamson, S.J., Romani, G.L., Kaufman, L., Modena, I. (1983). Biomagnetism. New York: Plenum Press.

[2] Vrba, J., Robinson, S.E. (2002). SQUID sensor array configurations for magnetoencephalography applications. Supercond. Sci. Technol., 15, R51-R89.

[3] Kötitz, R., Matz, H., Trahms, L., Koch, H., Weitschies, W., Rheinländer, T., Semmler, W., Bunte, T. (1997). SQUID based remanence measurements for immunoassays. IEEE Trans. Appl. Supercond., 7, 3678-3681. 